\title{
A Novel Video-Vibration Monitoring System for Walking Pattern Identification on Floors
}

\author{
Osama Abdeljaber ${ }^{1}$, Mohammed Hussein $^{2}$, Onur Avci $^{3}$, Brad Davis $^{4}$, Paul Reynolds ${ }^{5}$ \\ ${ }^{1}$ Research Assistant, Department of Civil and Architectural Engineering, Qatar University, \\ Doha, Qatar. Email: o.abdeljaber@qu.edu.qa (corresponding author) \\ ${ }^{2}$ Associate Professor, Department of Civil and Architectural Engineering, Qatar University, \\ Doha, Qatar. Email: mhussein@qu.edu.qa \\ ${ }^{3}$ Former Assistant Professor, Department of Civil and Architectural Engineering, Qatar \\ University, Doha, Qatar. Email: oavci@vt.edu \\ ${ }^{4}$ Associate Professor, Department of Civil Engineering, University of Kentucky, 373 Raymond \\ Building, Lexington, KY 40506, USA. Email: dbraddavis@uky.edu \\ ${ }^{5}$ Professor of Structural Dynamics and Control, Mathematics, and Physical Sciences, College of \\ Engineering, University of Exeter, North Park Road, Exeter, EX4 4QF, UK. Email: \\ p.reynolds@exeter.ac.uk
}

\begin{abstract}
Walking-induced loads on office floors can generate unwanted vibrations. The current multiperson loading models are limited since they do not take into account nondeterministic factors such as pacing rates, walking paths, obstacles in walking paths, busyness of floors, stride lengths, and interactions among the occupants. This study proposes a novel video-vibration monitoring system to investigate the complex human walking patterns on floors. The system is capable of capturing occupant movements on the floor with cameras, and extracting walking trajectories using image processing techniques. To demonstrate its capabilities, the system was installed on a real office floor and resulting trajectories were statistically analyzed to identify the actual walking patterns, paths, pacing rates, and busyness of the floor with respect to time. The correlation between the vibration levels measured by the wireless sensors and the trajectories extracted from the video recordings were also investigated. The results showed that the proposed video-vibration monitoring system has strong potential to be used in training data-driven crowd models, which can be used in future studies to generate realistic multi-person loading scenarios.
\end{abstract}

Keywords: Human walking patterns; vibration serviceability; video monitoring; vibration monitoring; video-vibration monitoring; probabilistic occupancy map algorithm; walking trajectory extraction; homography computations; occupant induced floor vibrations. 


\section{Introduction}

Advancements in construction materials have enabled engineers and architects to design with light-weight and high-strength structural materials, which often result in slender structural members [1]. That, with the trend toward open office spaces with few full-height partitions and lightweight furniture, has resulted in flexible and lightly damped floor systems that are susceptible to annoying vibrations due to human excitations [2-4]. With lower natural frequencies and damping ratios of floors, the number of complaints by occupants has increased over the years and vibration serviceability has become an important limit state for the design of floor systems $[5,6]$.

It is noted that floor live loads used in serviceability evaluations differ from code-prescribed live loads used in strength and stiffness evaluations. The former are best estimates of live loads in place during a vibration event whereas the latter are much higher values based on long recurrence intervals, such as the 50 year interval used in ASCE (2017) [7]. Due to the possibility of high loads at some time in the service life of a structure, the latter have not changed due to the advent of lighter office furnishings.

In residential floors, excessive vibrations cause occupant discomfort and irritation, while for office floors, the discomfort results in a loss of focus, creativity and productivity in the work place [8-10]. For human well-being, researchers have been studying floor vibrations serviceability over the last three decades to come up with efficient ways to keep the vibration levels below tolerance limits for different types of floors [11-13]. The findings of these studies have been implemented in a number of structural design guides [14-17].

For vibration serviceability assessment of floors, researchers have often focused on modeling of the load applied to the floor structure $[18,19]$. The earlier load models were based on vibrations 
due to a single person [20]. Later, researchers have been focusing on load models with more than one occupant (which has not been reflected in the design guides yet). Such multi-person load models are more realistic especially when evaluating the vibration serviceability of office floors [21].

An ideal load model should incorporate several parameters such as walking paths, pacing rates, number of walkers, and busyness of the floor. Since such models are not available [22], the current design guidelines such as [23-26] require evaluating vibration serviceability for singleperson walking scenarios only. Živanović et al. [22] indicated that the single person walking approach results in inaccurate estimates of floor vibrations. This is a crucial finding clearly supporting the need for the study presented in this paper.

The objective of the research described herein is to develop a method of acquiring information on the actual walking behavior on an office floor. With such a method, researchers will be able to gather data that would result in more realistic load models to be implemented in future design guides. More realistic load models would eventually result in more accurate assessment of floor vibration serviceability.

The objective was accomplished by the following tasks:

- Conduct continuous in-service video-vibration monitoring of an office floor during a work day.

- Use occupant tracking and detection algorithms to extract the walking trajectories from the recorded videos.

- Investigate the walking behavior and patterns on the monitored floor and analyze the extracted trajectories. 
- Examine the correlation between the extracted walking path and the vibration response of the floor.

The novelty of the work carried out in this paper lies in the fact that it integrates a trajectory extraction technique with vibration measurements to develop a system for simultaneous videovibration monitoring. The information extracted by this system can be used in future studies to create an accurate model for multi-person loading scenarios which can be incorporated into future design guidelines by floor vibration serviceability researchers. The outcome of this research will pave the way for improving the current models used in vibrations serviceability assessment of floors.

\section{Descriptions of Specimen, Equipment and the Video Monitoring System}

The temporary offices of Qatar Rail Company (located in Doha, Qatar) were used for the inservice video monitoring demonstration. The area is $15.6 \mathrm{~m}$ by $7.5 \mathrm{~m}$ in plan and hosts 16 desks distributed along three corridors (Figures 1 and 2). The floor consists of $15 \mathrm{~mm}$ thick cement boards attached to the top chord of the aluminum floor trusses, as shown in Figure 3 . The monitoring exercise was conducted continuously from 7:00 AM to 5:30 PM to encompass an entire workday. 


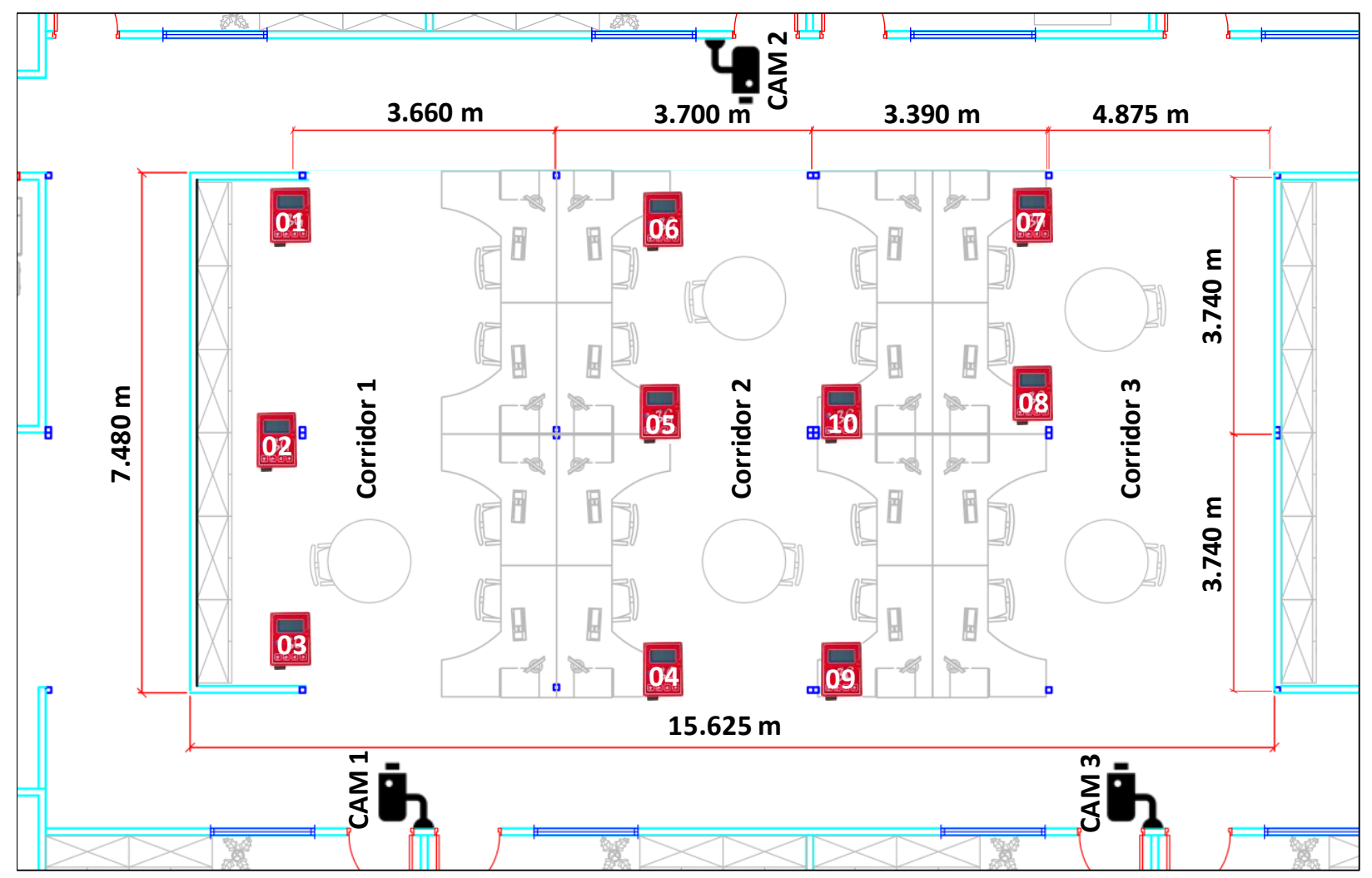

Figure 1. Plan view of the monitored workplace showing the locations of cameras and sensors.

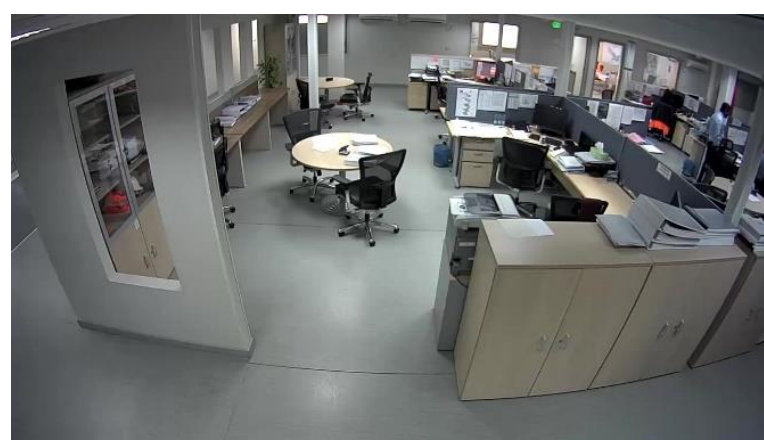

(a)

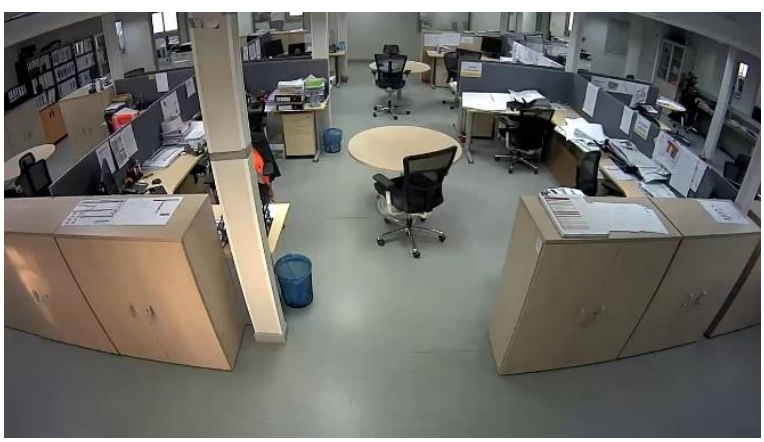

(b)

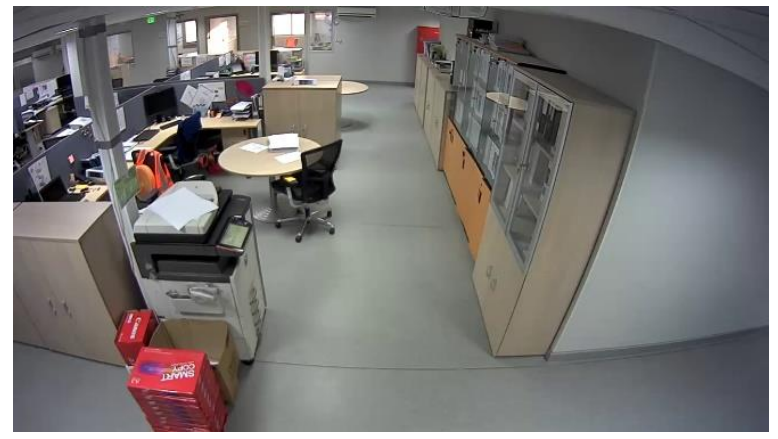

(c)

Figure 2. Views of the monitored floor: (a) Corridor 1. (b) Corridor 2. (c) Corridor 3. 


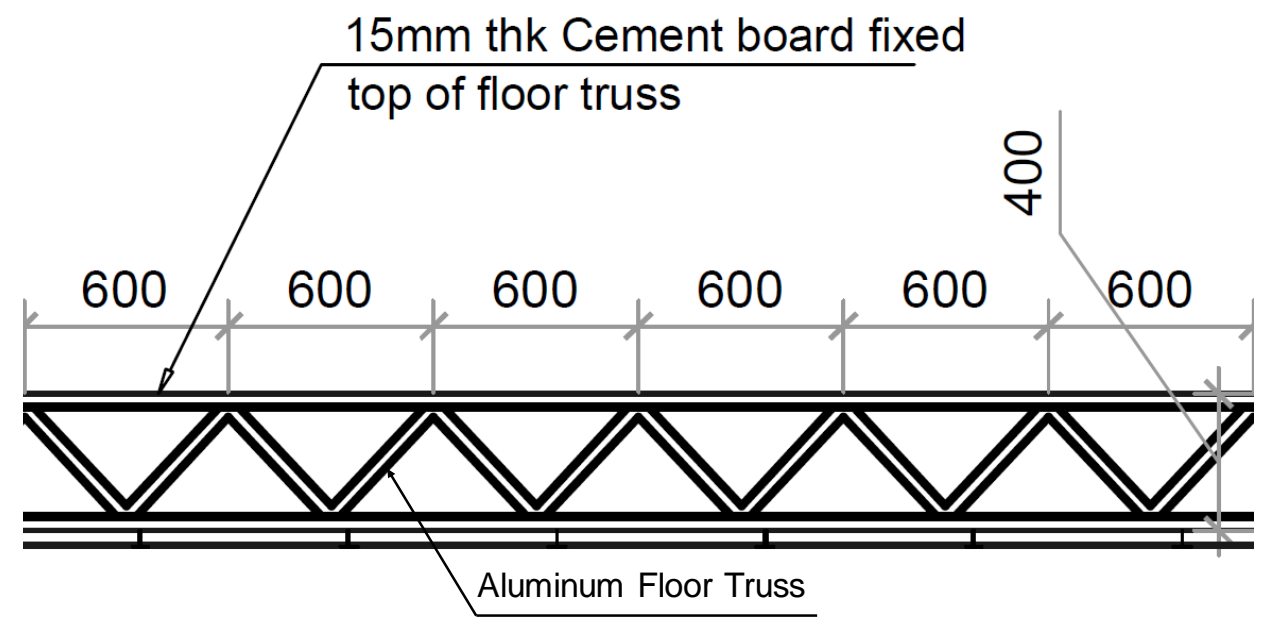

Figure 3. Elevation view of the floor (all dimensions in $\mathrm{mm}$ )

Three Samsung SNH-P6410BN cameras (Figure 4), denoted CAM1, CAM2, and CAM3 in Figure 1 were used to monitor the three corridors. Each camera was equipped with a 32 Gigabyte SD card to store the recordings. All videos were recorded in high definition (HD) resolution $(1280 \times 720$ pixels) and a frame rate of 15 frames per second (fps). The cameras were operated using the SmartCam mobile application.
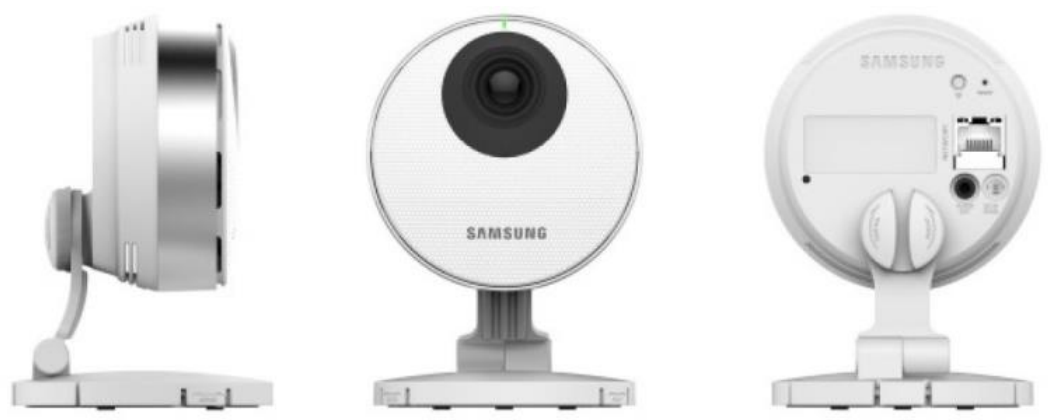

Figure 4. Samsung SNH-P6410BN camera

Simultaneous to video monitoring, vertical floor accelerations at the ten locations indicated in Figure 1 were recorded using TROMINO ${ }^{\circledR}$ ENGY wireless sensing units (Figure 5). TROMINO units are triaxial sensors having 3 velocimetric and 3 accelerometric channels. These sensors are ultra-compact and light-weight which makes them ideal for stratigraphic applications, modal 
testing, and vibration serviceability assessment. The sampling frequency was set to $128 \mathrm{~Hz}$. A representative velocity time-history measured by one of the wireless units is presented in Figure 6. The reader is referred to [27] for the specifications and to [28] for an example study conducted with of these sensors.

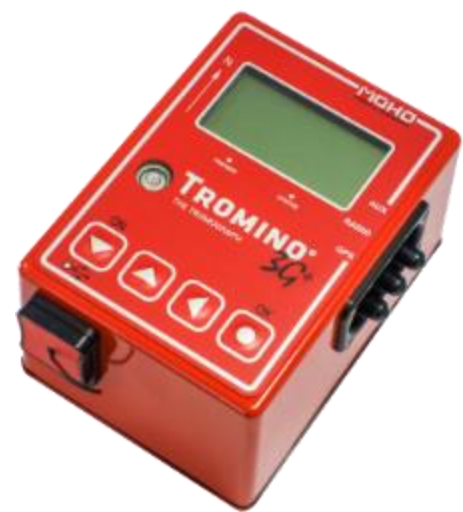

Figure 5. TROMINO ${ }^{\circledR}$ ENGY wireless sensing unit.

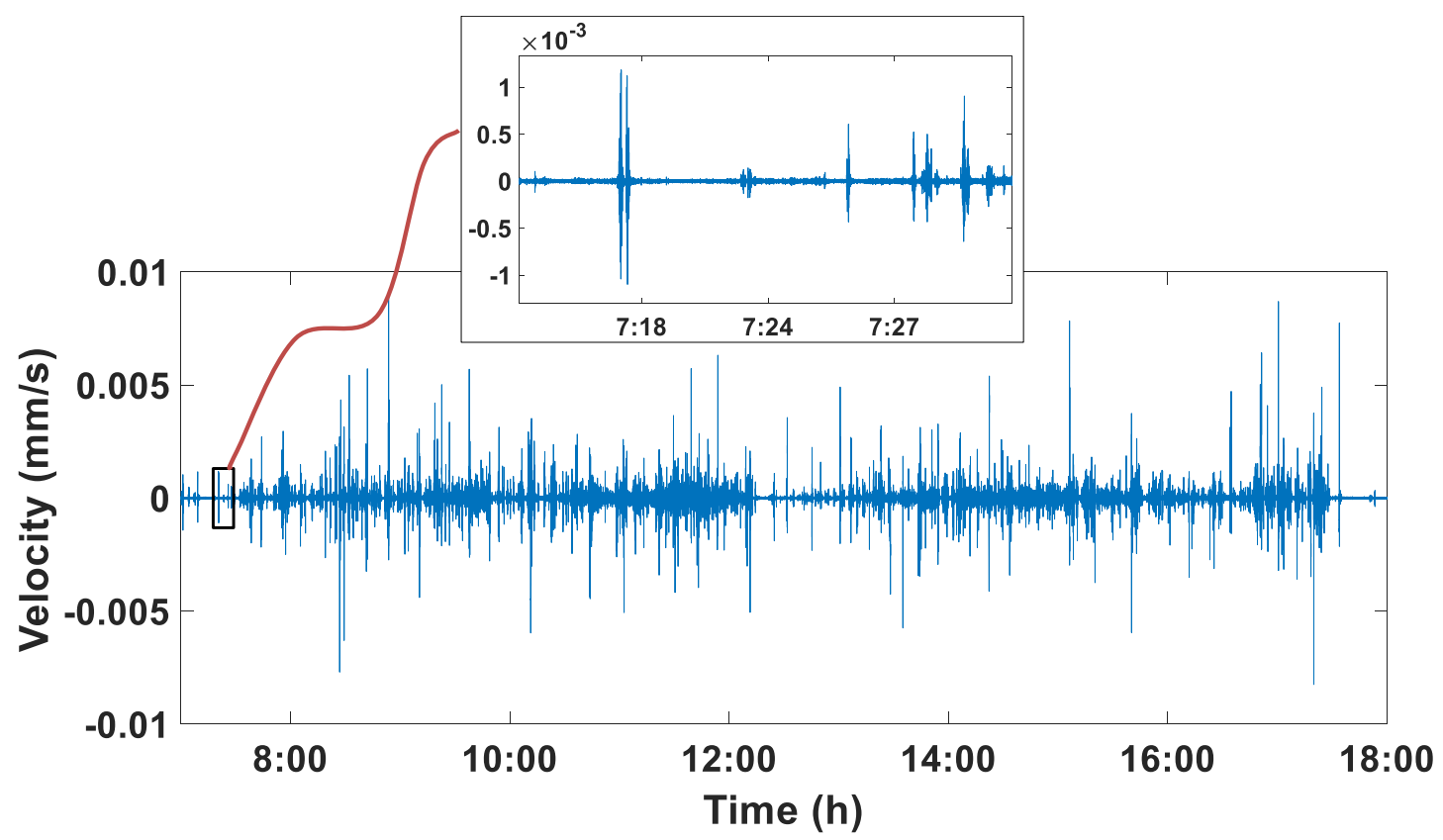

Figure 6. A representative velocity time-history recorded by one of the wireless units. 
The wireless sensors have the option to be synchronized with radio signals or a GPS time marker. Even though the GPS transmitters of the sensors have been successfully used by the authors on an open structure (e.g. a stadium structure); the sensors did not function properly indoors (for the floor of the structure used in this study). Therefore, the authors had to operate with "radio synchronization" instead of "GPS synchronization" for the sensors, on the floor structure. It is found that due to the signal attenuation caused by construction materials, the satellite based GPS signals lose considerable power while indoors since the host structure negatively affects the required coverage for receivers by at least four satellites. Furthermore, the multiple reflections at the housing structure cause multi-path propagation resulting in uncontrollable errors with the units. As such, it is widely reported that the waves are attenuated and scattered by roofs and walls of the structures, which means the GPS is not suitable for indoor use. Even with the latest technology chips, it is reported that the resulting location is not accurate enough to be useful to operate with as the position of the device cannot be pinpointed precisely.

\section{Extraction of Walking Trajectories}

The videos recorded by the three cameras were processed to extract the trajectories that represent the occupant movement across the three corridors over time. The first step was reducing the resolution to $640 \times 360$ pixels to minimize the computational time and effort required to process each frame. After that, the videos were corrected by removing the lens distortion associated with the use of wide-angle cameras. To obtain the intrinsic parameters of the camera required for the undistortion of the videos, several photos of a checkerboard are captured by the camera from various angles and locations as shown in Figure 7. The photos were processed with Matlab Camera Calibration Toolbox [29] and the resulting parameters were obtained and summarized in 
Table 1. All video recordings were undistorted according to the extracted camera parameters using "undistortImage()" function in Matlab. This function simply returns an image with lens distortion removed and also returns the location of the output image origin which is set in terms of the input intrinsic coordinates specified in camera parameters [29]. An example for the undistortion process is shown in Figure 8.
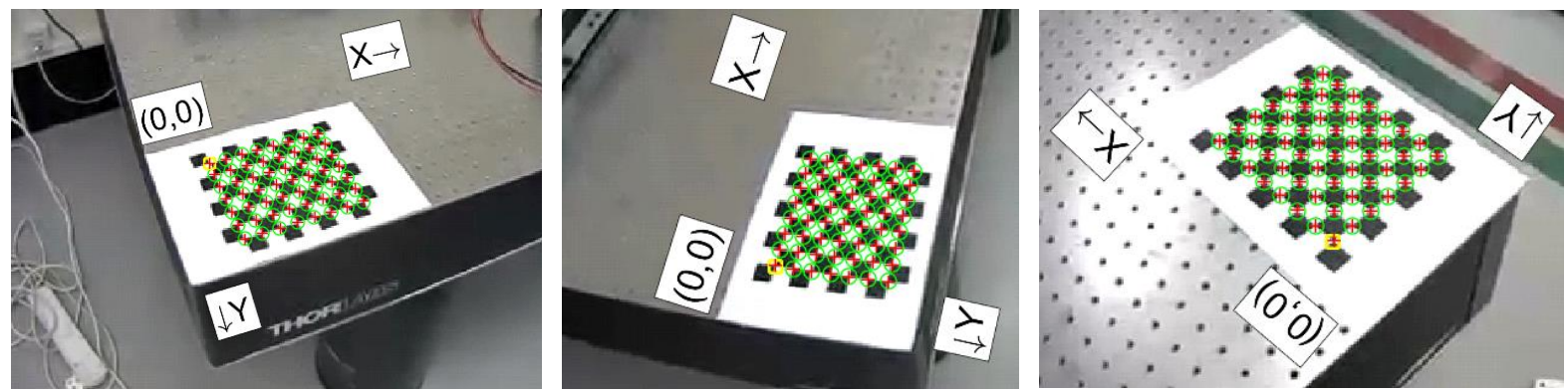

Figure 7. Checkerboard images used to obtain the intrinsic parameters of the camera.

Table 1. Intrinsic parameters of the camera obtained by Matlab Camera Calibration Toolbox.

\begin{tabular}{ll}
\hline Property & Value \\
\hline Focal length in pixels $\left(f_{x}, f_{y}\right)$ & $347.7,347.03$ \\
Optical center in pixels $\left(c_{x}, c_{y}\right)$ & $302.7,170.2$ \\
Radial distortion parameters $\left(r_{x}, r_{y}\right)$ & $-0.3222,0.1072$ \\
Skew Coefficient $(s)$ & 0 \\
\hline
\end{tabular}

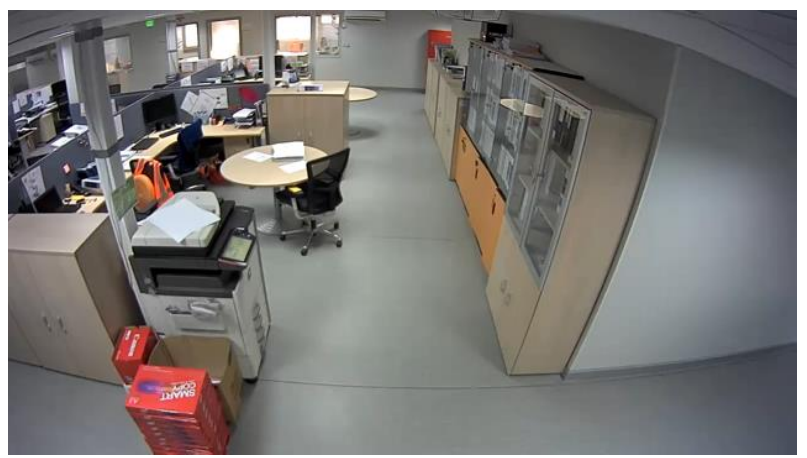

(a)

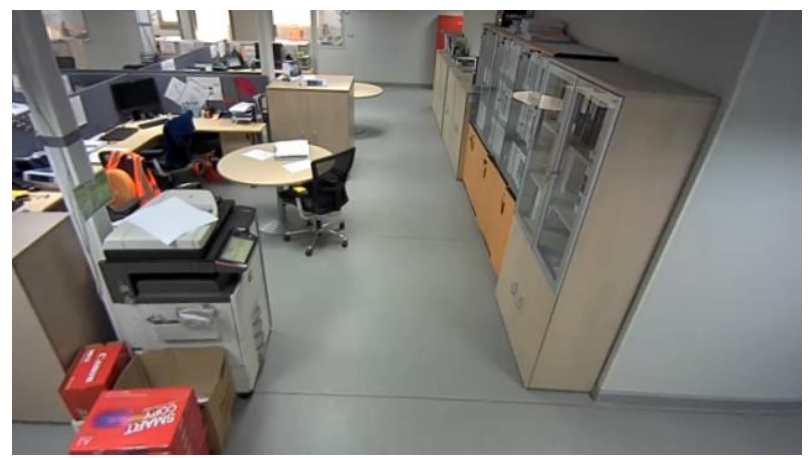

(b)

Figure 8. A video frame capture by CAM3. (a) Before undistorting. (b) After undistorting. 
As discussed later, the Probabilistic Occupancy Map Algorithm (POM) [30] is used in this work to determine the locations of the occupants in each video frame. Introduced by Fleuret et al. [30], the POM algorithm was later used in follow-up studies [31-36]. The POM algorithm requires segmenting the view of each camera into a number of rectangles that represent human silhouettes with a height of $H$ and a width of $W$ (an example is shown in Figure 9).

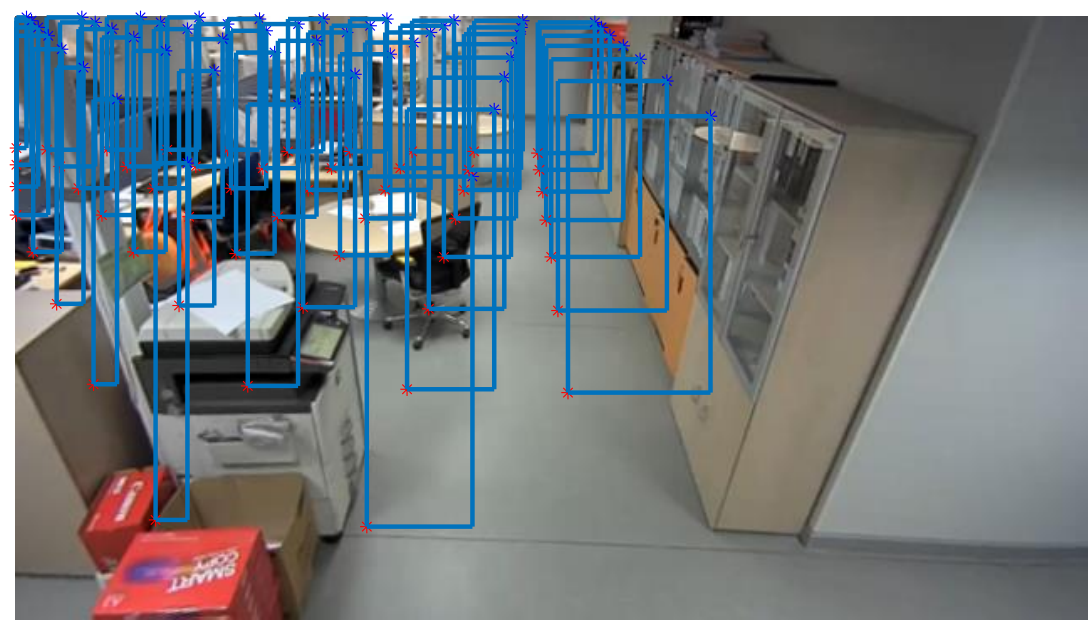

Figure 9. Example of rectangles (silhouettes) that represent possible occupant locations as viewed by CAM3. A large mesh size (small number of rectangles) was used here for clarity.

To construct these rectangles, it is required to define a grid that represents the possible occupant locations in world coordinates ( $\mathrm{x}$ and $\mathrm{y}$ ). Then, for each camera, two homography matrices $\mathbf{H}_{G, c=i}$ and $\mathbf{H}_{T, c=i}$ are obtained and used to project this grid to the ground $(z=0)$ and the head $(z=H)$ planes of the camera view. To calculate the matrix $\mathbf{H}_{G, c=i}$, which is the homography matrix of the $i^{\text {th }}$ camera corresponding to the ground plane, the matrix $\mathbf{M}$ is defined as [37]:

$$
\mathbf{M}=\left[\begin{array}{ccccccccc}
-x_{1, G, c_{i}} & -y_{1, G, c_{i}} & -1 & 0 & 0 & 0 & x_{1, G, c_{i}} x_{w, 1} & y_{1, G, c_{i}} x_{w, 1} & x_{w, 1} \\
0 & 0 & 0 & x_{1, G, c_{i}} & -y_{1, G, c_{i}} & -1 & x_{1, G, c_{i}} y_{w, 1} & y_{1, G, c_{i}} y_{w, 1} & y_{w, 1} \\
\vdots & \vdots & \vdots & \vdots & \vdots & \vdots & \vdots & \vdots & \vdots \\
-x_{4, G, c_{i}} & -y_{4, G, c_{i}} & -1 & 0 & 0 & 0 & x_{4, G, c_{i}} x_{w, 4} & y_{4, G, c_{i}} x_{w, 4} & x_{w, 4} \\
0 & 0 & 0 & x_{4, G, c_{i}} & -y_{4, G, c_{i}} & -1 & x_{4, G, c_{i}} y_{w, 4} & y_{4, G, c_{i}} y_{w, 4} & y_{w, 4}
\end{array}\right]
$$


where $\left(x_{w, 1}, y_{w, 1}\right), \ldots,\left(x_{w, 4}, y_{w, 4}\right)$ are the world coordinates of any noncolinear points in the grid, $\left(x_{1, G, c_{i}}, y_{1, G, c_{i}}\right), \ldots,\left(x_{4, G, c_{i}}, y_{4, G, c_{i}}\right)$ are the camera coordinates (in pixels) of the same four points in the ground plane of the $i^{\text {th }}$ camera view. The matrix $\mathbf{H}_{G, c=i}$ is then calculated as the first eigenvector (reshaped into a $3 \times 3$ matrix) of $\mathbf{M}^{\mathbf{T}} \mathbf{M}$. Also, the matrix $\mathbf{H}_{T, c=i}$ is calculated in a similar way except the camera coordinates of the four points in the head plane of the $i^{\text {th }}$ camera view $\left(x_{1, T, c_{i}}, y_{1, T, c_{i}}\right), \ldots,\left(x_{4, T, c_{i}}, y_{4, T, c_{i}}\right)$ are used to compute $\mathbf{M}$.

As shown in Figure 10, to calculate $\mathbf{H}_{G, c=i}$ of the three cameras used in this work, each camera was used to take a picture of a $120 \times 60 \mathrm{~cm}$ tabletop placed on the ground at a known location. The four points required for homography computations were taken as the four corners of the tabletop (after eliminating lens distortion, as explained earlier). Similarly, as shown in Figure 11, to compute $\mathbf{H}_{T, c=i}$ of the three cameras, each camera was used to take a picture of a $120 \times 60 \mathrm{~cm}$ tabletop placed at $H=175 \mathrm{~cm}$ above the ground at a known location. Note that $H$ was taken as $175 \mathrm{~cm}$ to represent the height of an average human. The four points required for homography computations were taken as the four corners of the tabletop (after undistorting the picture). After the homography matrices were computed, it was possible to project a $20 \times 20 \mathrm{~cm}$ mesh to the ground and head planes of each camera view as shown in Figures 10 and 11. 


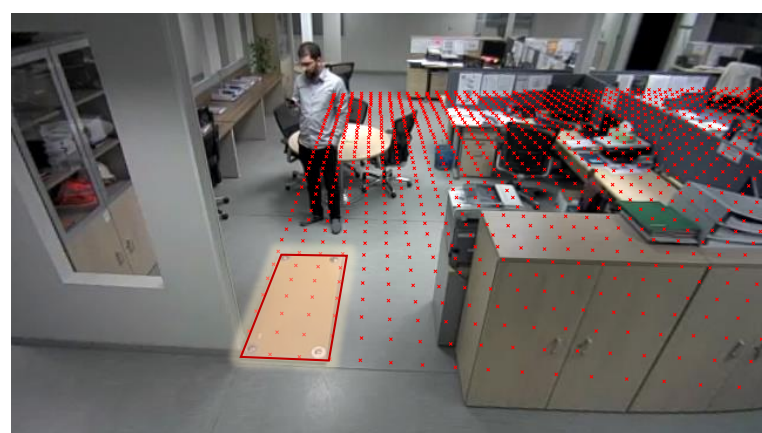

(a)

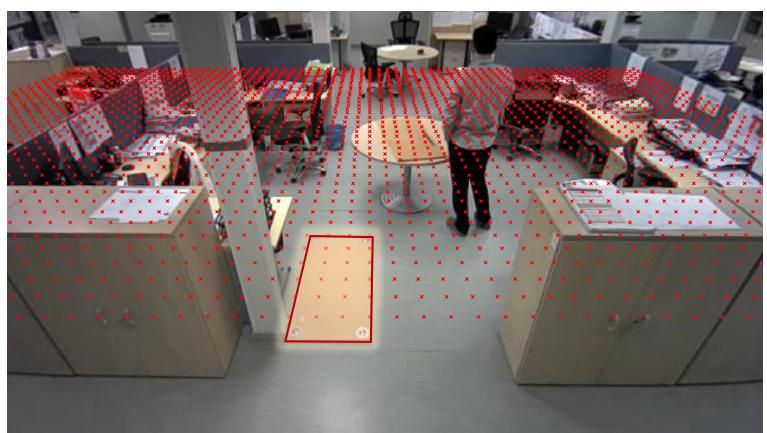

(b)

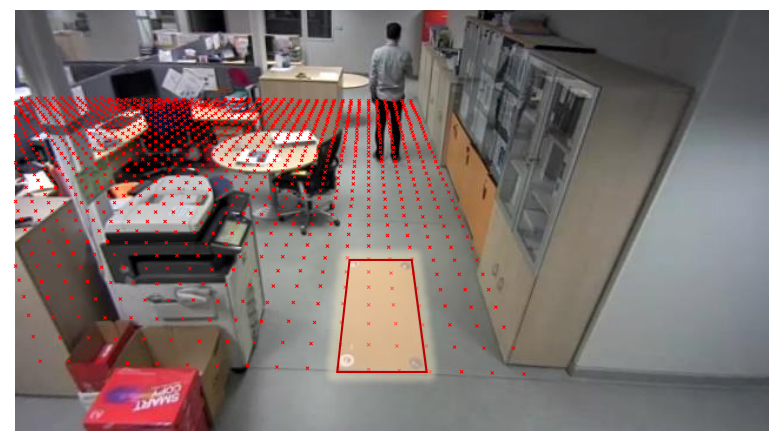

(c)

Figure 10. A grid with a mesh size of $20 \times 20 \mathrm{~cm}$ projected to the ground plane $(\mathrm{z}=0)$ as viewed by: (a) CAM1. (b) CAM2. (c) CAM3.

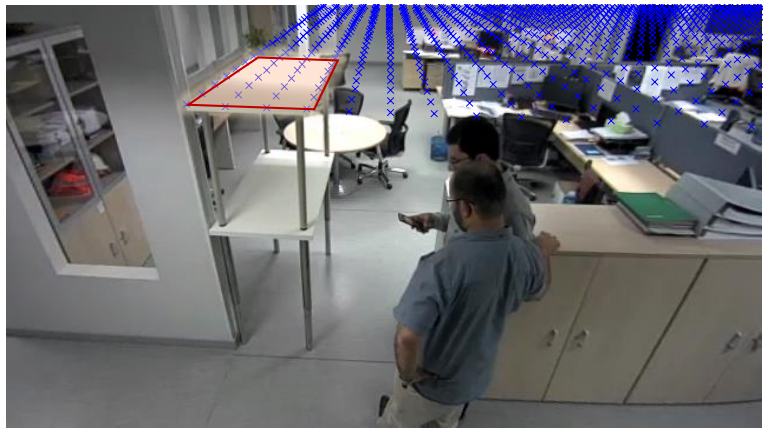

(a)

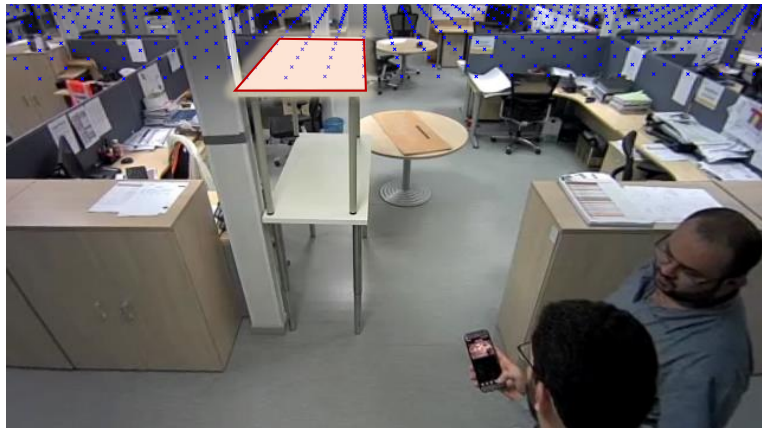

(b)

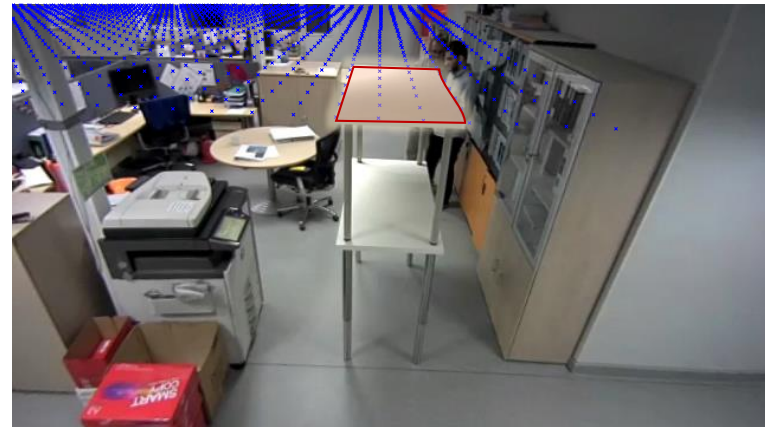

(c)

Figure 11. A grid with a mesh size of $20 \times 20 \mathrm{~cm}$ projected to the head plane $(\mathrm{z}=175 \mathrm{~cm})$ as viewed by: (a) CAM1. (b) CAM2. (c) CAM3. 
As a next step, for each camera, a rectangle was defined for each location of the grid. The bottom corner of the $j^{\text {th }}$ rectangle $\left(r_{b, x, j}, r_{b, x, j}\right)$ was computed by transforming the corresponding grid point $\left(x_{w, j}, y_{w, j}\right)$ from world coordinates to camera coordinates as follows [37]:

$$
\left[\begin{array}{lll}
r_{b, x, j} & r_{b, y, j} & 1
\end{array}\right]^{\mathrm{T}}=\mathbf{H}_{G, c=i}^{-1}\left[\begin{array}{lll}
x_{w, j} & y_{w, j} & 1
\end{array}\right]^{\mathrm{T}}
$$

The top corner of the $j^{\text {th }}$ rectangle $\left(r_{t, x, j}, r_{t, x, j}\right)$ was computed by transforming the corresponding grid point $\left(x_{w, j}, y_{w, j}\right)$ from world coordinates to camera coordinates as follows [37]:

$$
\left[\begin{array}{lll}
r_{t, x, j} & r_{t, y, j} & 1
\end{array}\right]^{\mathrm{T}}=\mathbf{H}_{G, c=i}^{-1}\left[\begin{array}{lll}
x_{w, j}-w & y_{w, j} & 1
\end{array}\right]^{\mathrm{T}}
$$

The last step before processing the videos using the POM algorithm was to apply background removal in order to isolate the occupants from the surroundings. To do so, a Gaussian Mixturebased background removal algorithm [38] was applied on each frame of the undistorted videos. This algorithm is implemented in OpenCV 2.4 Python package under the function cv2.BackgroundSubtractorMOG2(). An example for the background removal process is shown in Figure 12.
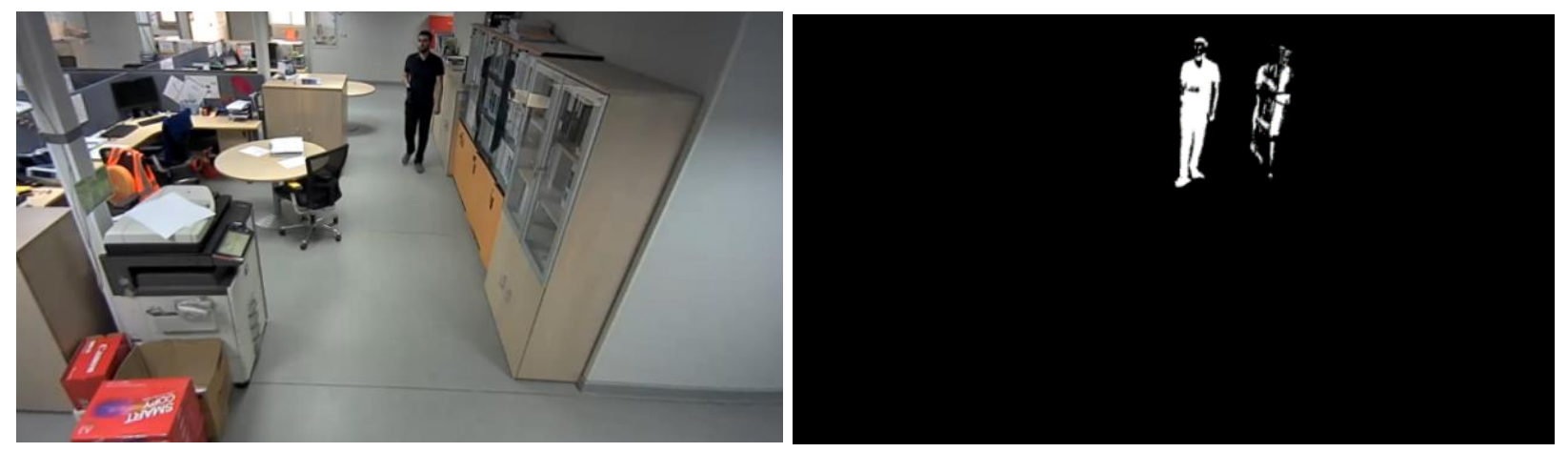

Figure 12. Background removal process. 
The POM algorithm was then applied to identify the locations of occupants at each time step. For each rectangle in the camera view, this algorithm computes the probability that this rectangle is occupied by a moving person. Next, for each frame, the algorithm constructs a Probabilistic Occupancy Map that represents the possible locations of occupants at the corresponding time step (an example is shown in Figure 13). Finally, the resulting maps were processed using a Kalman filter implemented in Matlab [29] to obtain the trajectories that represent the occupant movements on the monitored workplace over a given period of time. As an example, Figure 14 illustrates the ability of POM algorithm accompanied with the Kalman filter to detect and track an occupant. The trajectory extraction process explained in this section resulted in the 588 trajectories shown in Figure 15. Since the videos were captured at $15 \mathrm{fps}$, the sampling period of each trajectory was $1 / 15 \mathrm{sec}$.

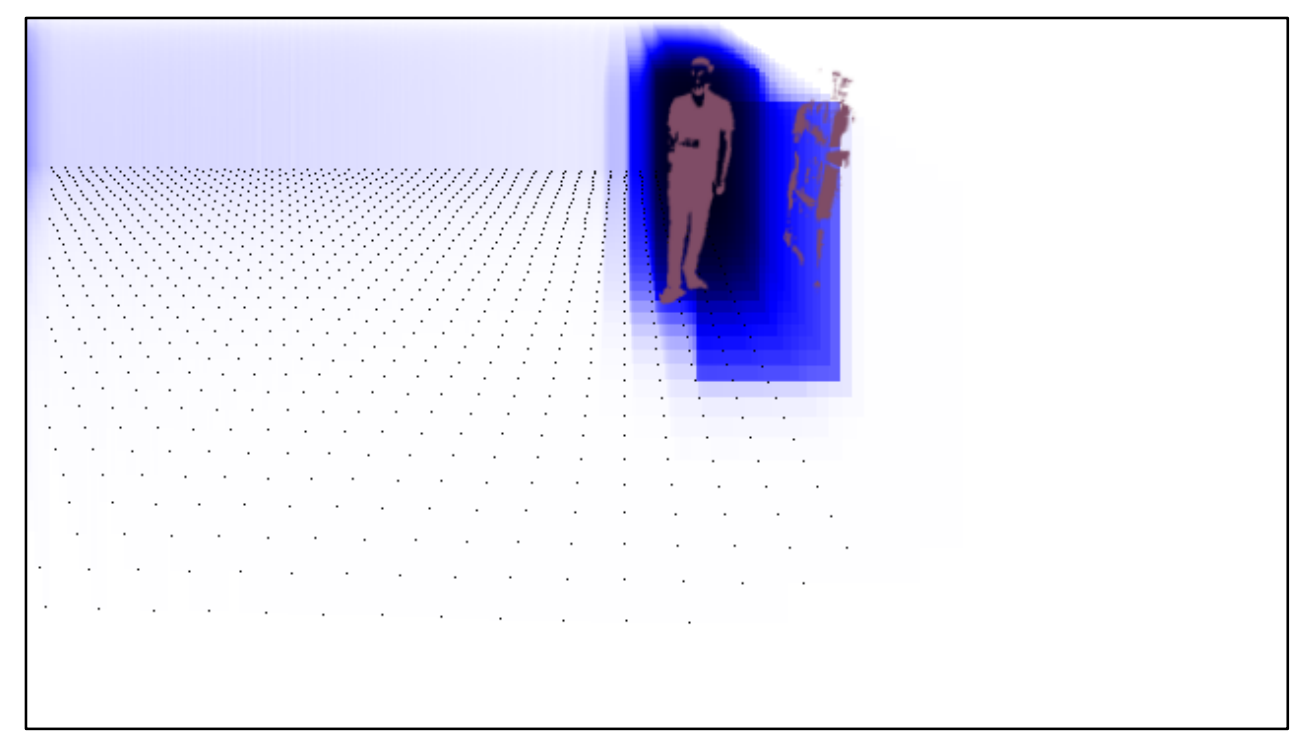

Figure 13. An example of the POM output. Darker colored rectangles represent locations with high probability of occupancy. 


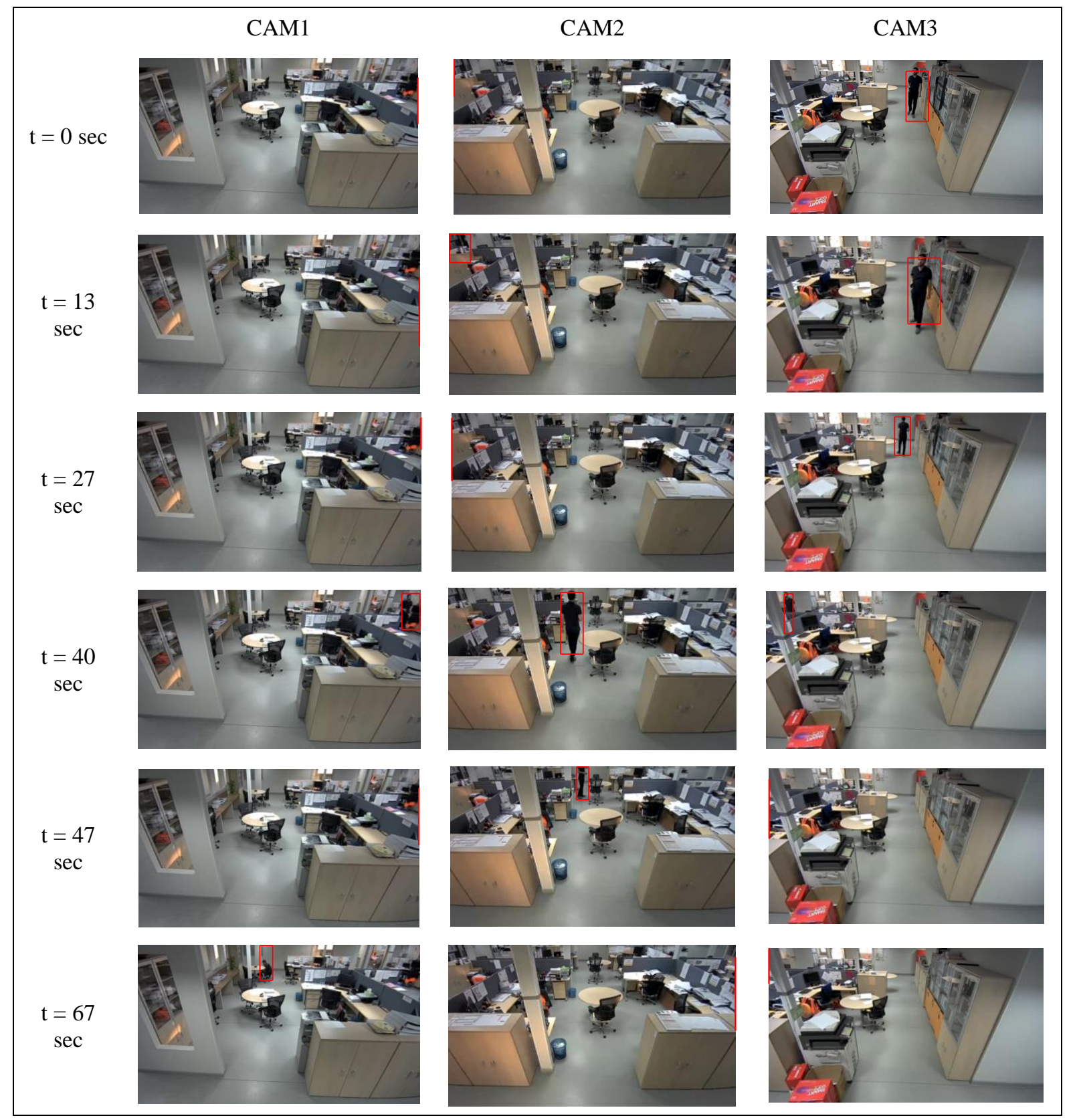

Figure 14. Detection and tracking of an occupant using POM algorithm and a Kalman filter. 


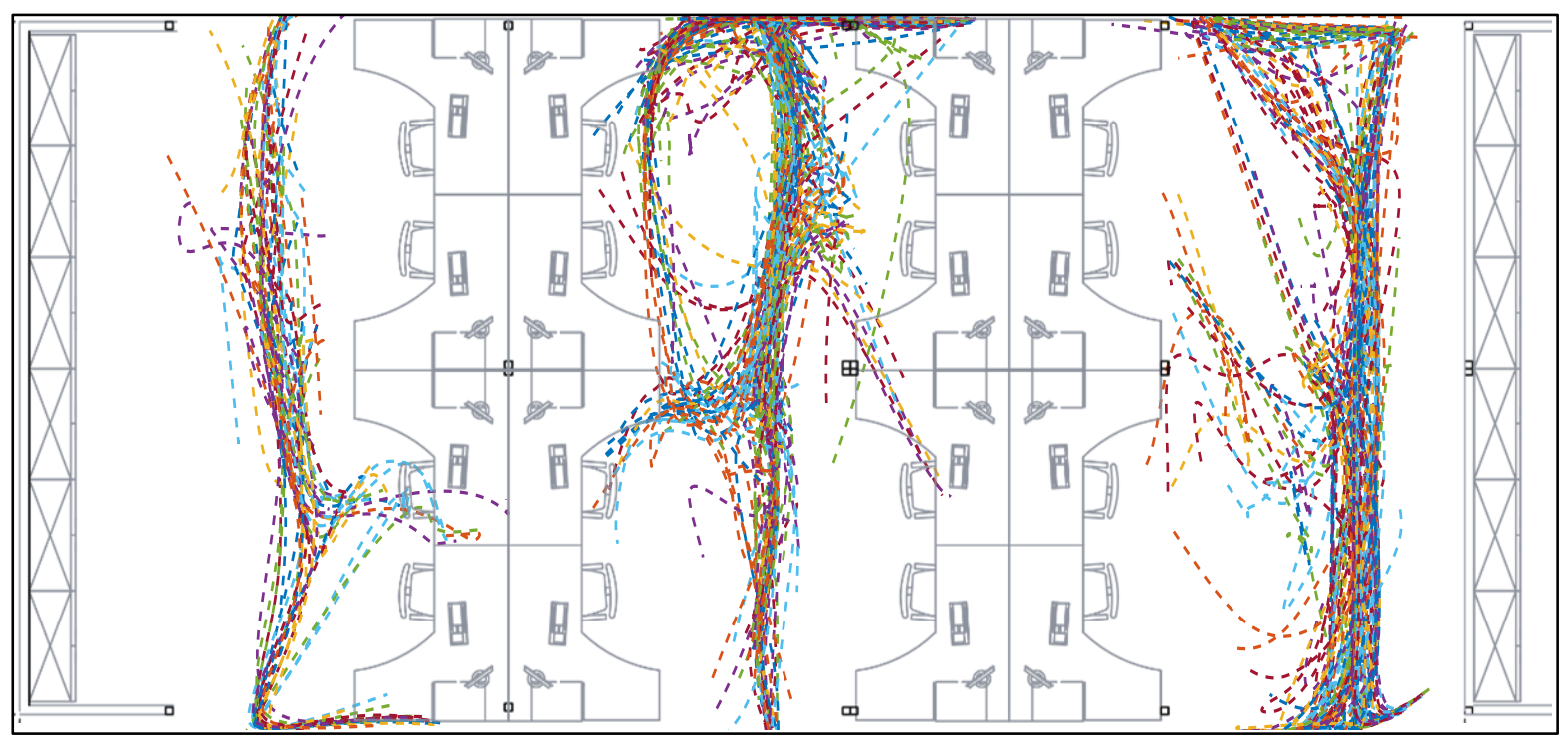

Figure 15. The extracted trajectories.

\section{Analysis of the Extracted Trajectories}

The 588 extracted trajectories shown in Figure 15 were analyzed to understand the occupant walking patterns on the floor. As an initial step, the trajectories were processed to produce a heat map so that the busyness of the monitored office floor can be studied. The heat map is shown in Figure 16. This figure is critical because the trajectory-based floor busyness analysis depicted in the figure will later be compared to the Vibration Dose Values (VDV) and Response Factors $(R)$ along the corridors to determine whether or not the results are consistent with the heat map.

In Figure 16, the color indicates the value of $\log _{10} N$, where $N$ is the number of times a moving person was detected at a specific location on the plan view. It is observed that the most active area of the floor is the entrance of corridor 2 (the North side of the corridor, pictorially). It is also observed that a lower level of activity was observed at corridor 1 . The level of activity in corridor 3 however is more than the activity on corridor 1 but less than the activity on corridor 2 . It is also observed that the furniture located in the corridors tend to restrict the movement of occupants by forcing them to walk around these obstacles to reach their destinations. This kind 
of restriction on the available walking space is an important parameter affecting the speed and trajectory of the occupants.

Considering the desk layout and the corridor arrangement shown in Figure 1, the relatively higher activity levels observed on corridor 2 (and corridor 3) can also be attributed to the duties of the office personnel stationed at the specific desk locations on the floor. For example, a project manager will typically have more conversations with other engineers and staff who walked to his/her desk which means there will be significant traffic from and to his/her desk. Similarly, a draftsman and engineers will be in constant communication and therefore would probably walk to each other's desks back and forth which would also result in busyness around certain desks.

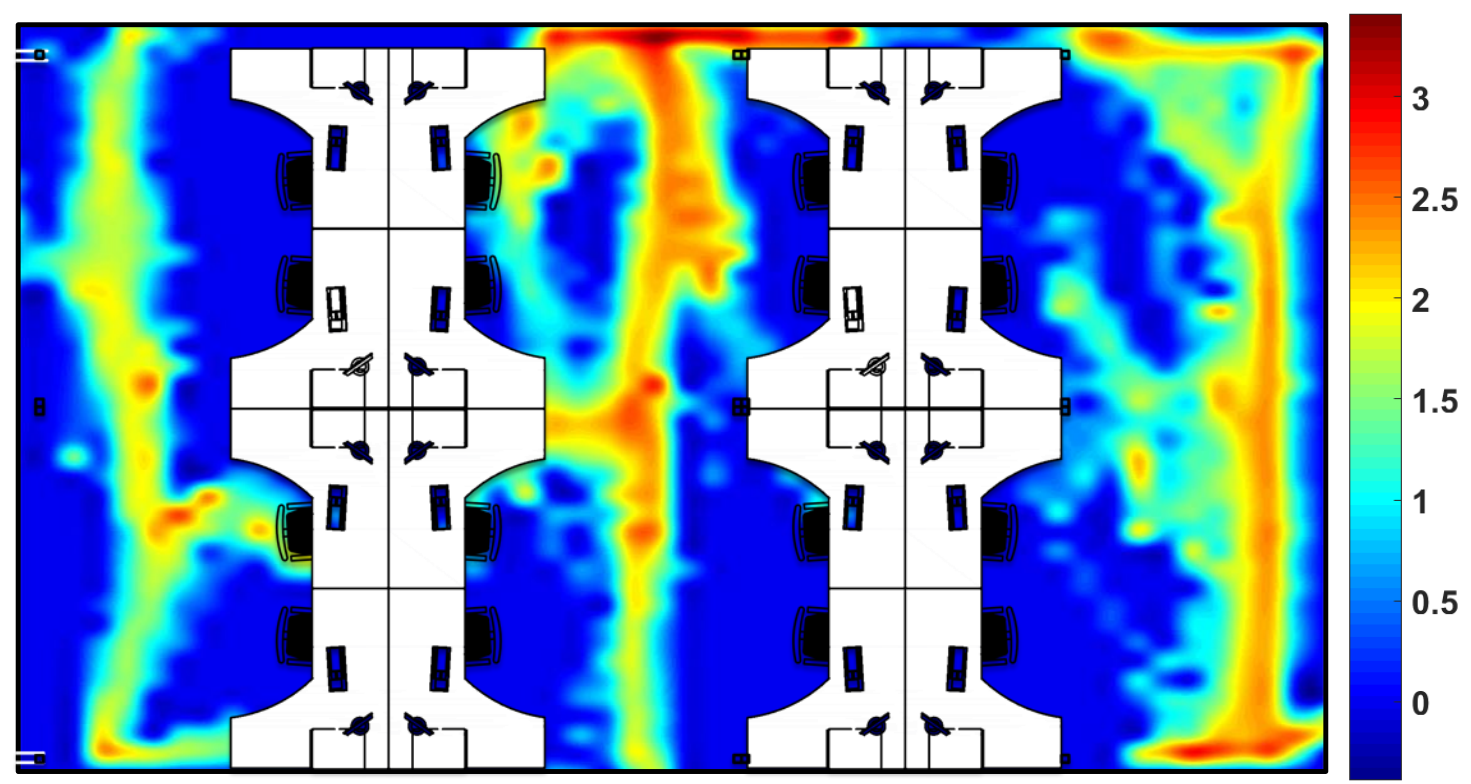

Figure 16. A heat map representing the busyness of the floor. The color in the color bar represents $\log _{10} N$, where $N$ is the number of times a moving person was detected at a particular area.

As a following step, statistical analyses were conducted to obtain in-depth information about the walking behavior of the occupants. The results of the analyses are summarized in Figure 17. The 
histogram shown in Figure 17a indicates that the highest level of activity (i.e. largest number of trajectories) occurred between 9:00 and 9:30 AM. Also, a significant drop in the level of activity occurred around the lunch break between 12:30 and 1:30 PM. The results shown in Figure 17a makes sense since 9:00 is the time where almost all of the occupants arrive at the office and reach their desk stations generating a lot of busyness in the floor traffic; similarly during the lunch break time the occupants tend to leave the floor and the number of trajectories drop.

According to the distribution of the total distances of the extracted trajectories shown in Figure $17 \mathrm{~b}$, the vast majority of the trajectories are shorter than $2.4 \mathrm{~m}$. Likewise, the distribution of displacements in Figure 17c indicates that the starting point of most of the trajectories is within $2 \mathrm{~m}$ of the ending point. Here it needs to be emphasized that the term "distance" is used for the total actual ground covered by the occupant while the term "displacement" is used with reference to a specific starting point. Considering the length of the corridors and the furniture (obstacles) on the way of occupants, both Figures $17 \mathrm{~b}$ and $17 \mathrm{c}$ also make sense.

In addition, Figure 17d indicates that the distribution of the average trajectory speed follows a normal distribution with a mean of $0.80 \mathrm{~m} / \mathrm{s}$. This relatively low walking speed is probably because the three corridors are narrow, which prevents the occupants from achieving higher pacing rates. The distribution of the instantaneous speeds across all trajectories (Figure 17e) reveals that the occupants often need to reduce their pacing rate below $0.25 \mathrm{~m} / \mathrm{s}$ to avoid obstacles, turn corners, or interact with colleagues. The observation made for Figure $17 \mathrm{e}$ is supported by Figure 17f which shows a histogram of the variation in trajectory speeds. This distribution shows that most of the trajectories have a significantly high coefficient of variation (COV) indicating that the occupants did not maintain a constant pacing rate in most cases. 


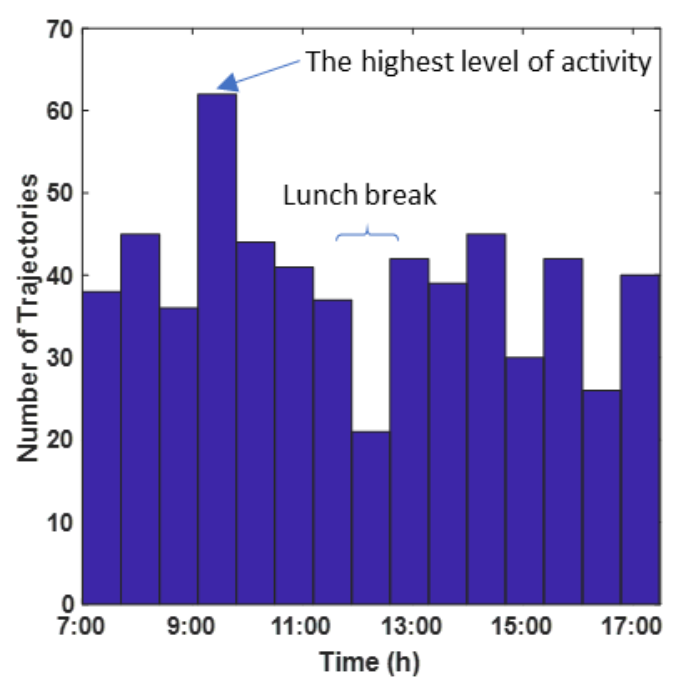

(a)

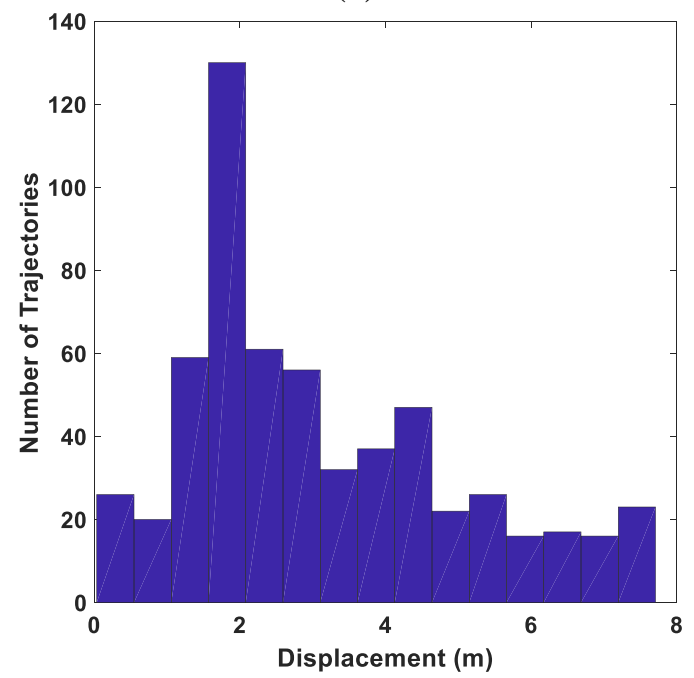

(c)

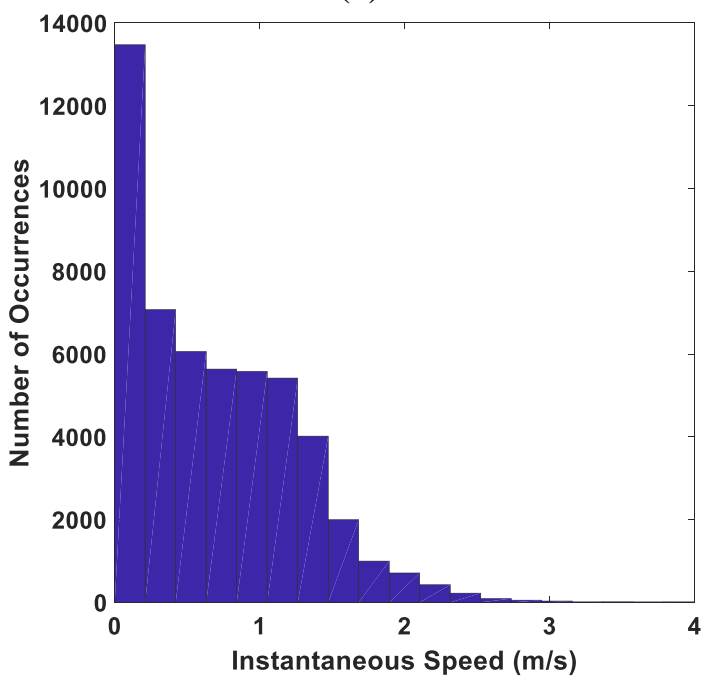

(e)

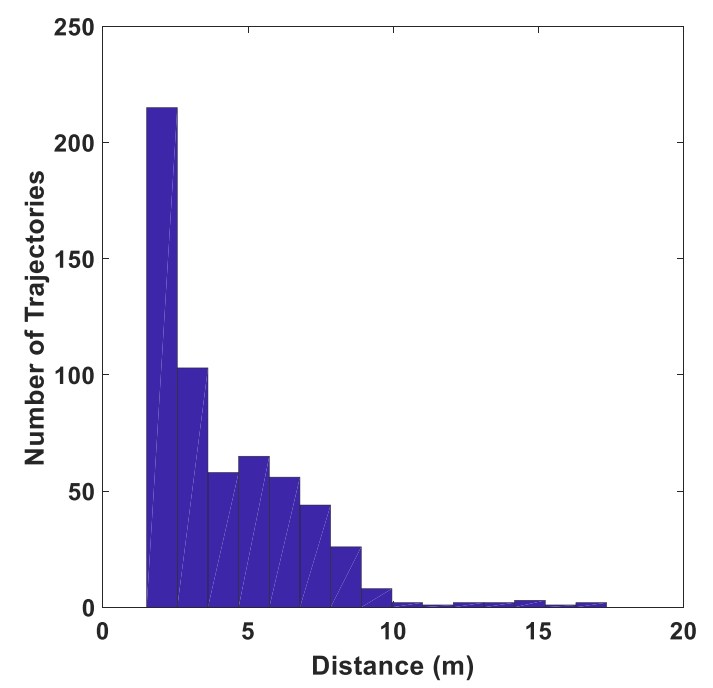

(b)

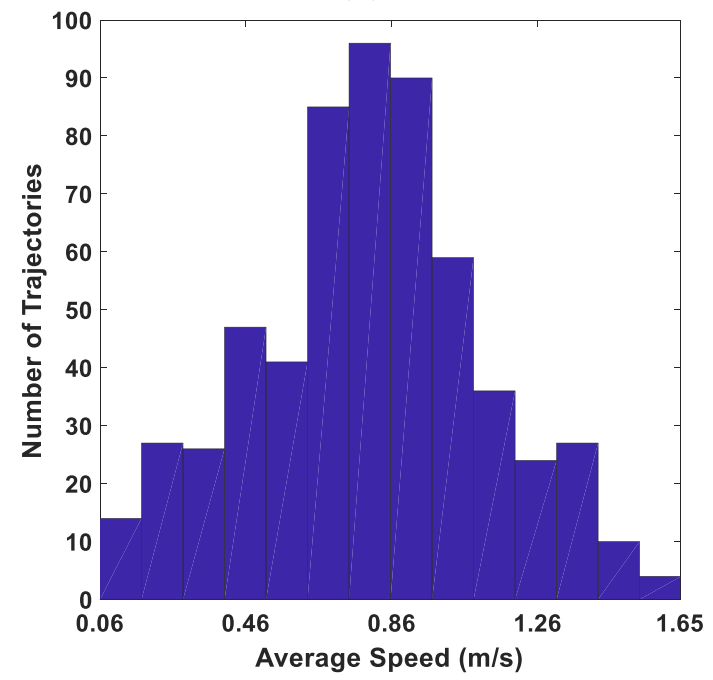

(d)

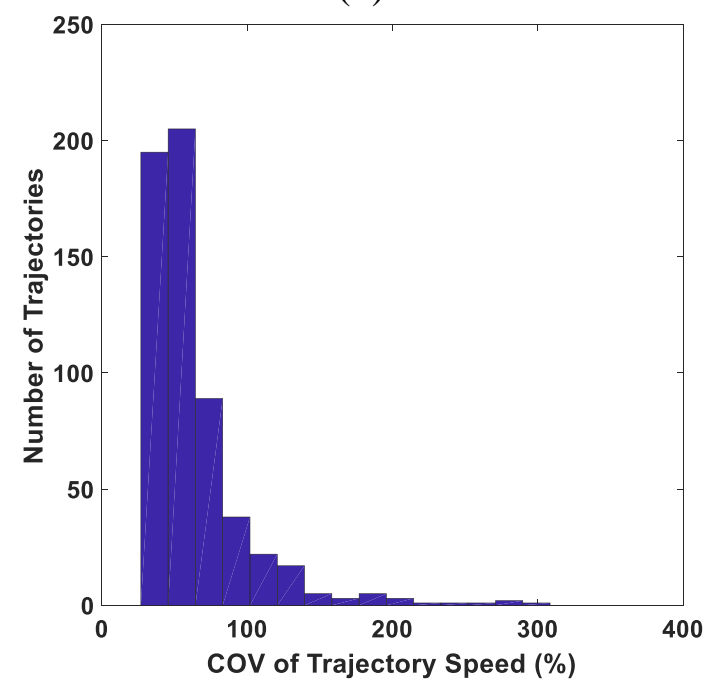

(f)

Figure 17. Statistical analysis results for the extracted trajectories. 
To test the consistency of the video and the vibration components of the proposed monitoring system, it is important to observe and examine the correlation between the trajectories extracted from video monitoring and the vibrations measured by the sensors. Therefore, the walking trajectory of a person passing through corridor 1 and the resulting vibration response at sensors 1, 2 and 3 (of corridor 1) are plotted in Figure 18. It should be noted that the y-axis quantity shown in Figure 18 are the normalized velocity response of the sensors, therefore it is a unitless quantity. As the person enters the corridor (Figure 18a), the maximum vibration amplitude can be observed around $t=2.1 \mathrm{~s}$ in the signal measured by sensor 3 (i.e. near the South entrance of the corridor, pictorially, per Figure 1). At $\mathrm{t}=4.3 \mathrm{~s}$ (Figure 18b), the extracted trajectory indicates that the person is passing through the middle of the corridor, and the corresponding signal measured by sensor 2 shows a distinctive spike as a result of the occupant walking excitation. Finally, as the person exits the corridor around $t=5.5 \mathrm{~s}$, the response of sensor 1 (i.e. near the North entrance of the corridor, pictorially, per Figure 1) exhibits significant fluctuation. This analysis reveals that the extracted trajectories and the acquired vibration measurements are in good agreement.
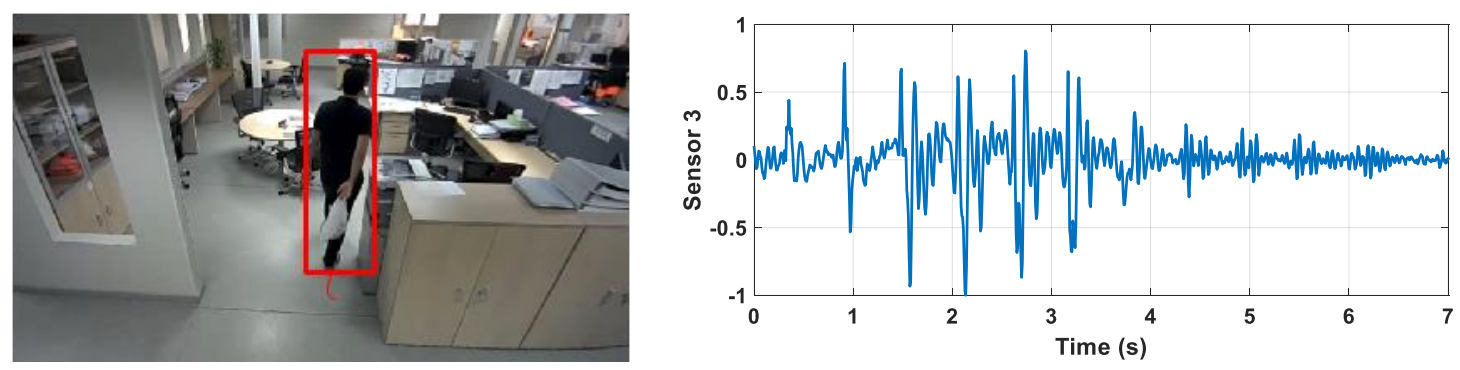

(a) 

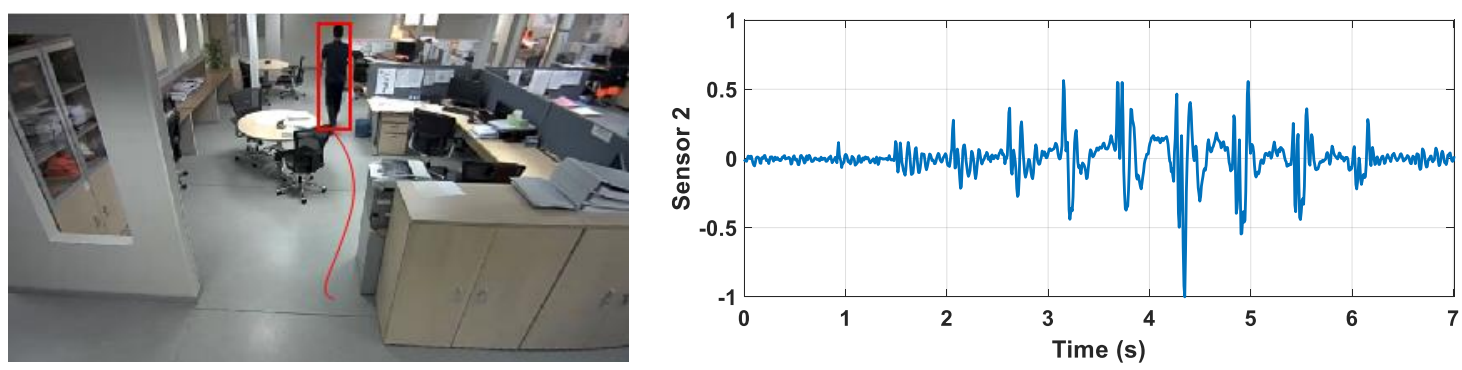

(b)
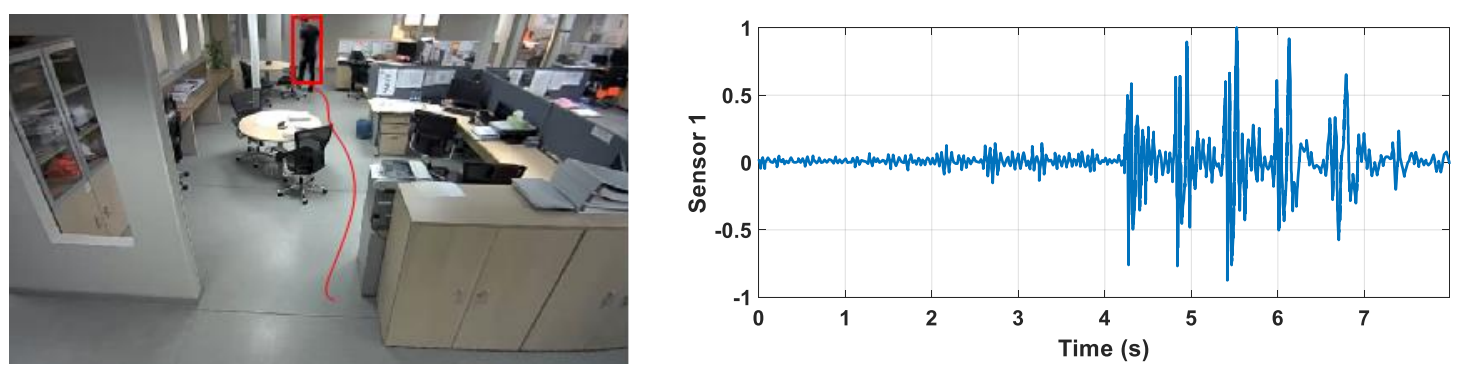

(c)

Figure 18. The normalized velocity response associated with a single walking trajectory at (a) sensor 1, (b) sensor 2, and (c) sensor 3.

The moving Root Mean Square (RMS) of the vibration response is shown for sensors 5 and 6, in Figure 19. The moving RMS was obtained by sliding a 1-hour window along the velocity signals of the sensors. In both Figure 19a and 19b, the highest RMS values are observed around 9:00 AM, while a significant drop in the vibration levels can be observed around 12:30 and 1:30 PM (i.e. the lunch break). This is a similar behavior observed in the histogram Figure 17a which revealed that the largest number of trajectories occurred (from 9:00 to 9:30 AM); and a significant drop in the level of activity occurred during the lunch break (from 12:30 to 1:30 PM). This shows that the vibration response measured by the wireless sensors is in agreement with the levels of activity identified by trajectory analysis which is a product of video monitoring. This consistency observed in Figure 19 and Figure 17a confirms the success of the individual video and vibration components of the monitoring system. 


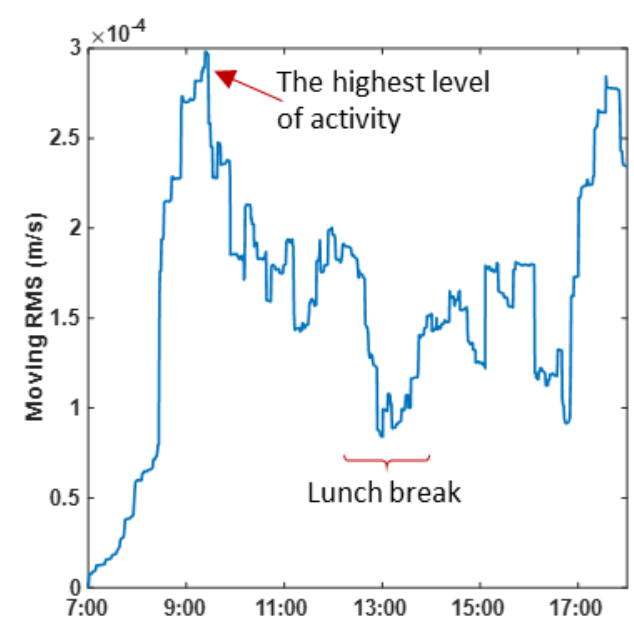

(a)

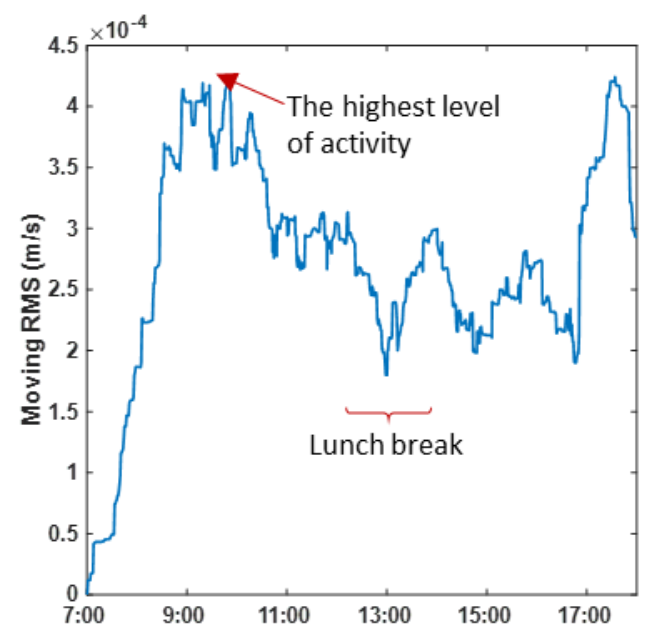

(b)

Figure 19. Moving RMS of the vibration response in terms of velocity at: (a) Sensor 5. (b) Sensor 6.

The Vibration Dose Values (VDV) at the ten sensor locations are shown in Figure 20. VDV is a cumulative measure of vibration exposure over a certain period that is commonly used for vibration serviceability assessment of office floors [20]. The VDV values were computed according to BS 6472-1:2008 [39] (Eq. 4) and the vibration signals in terms of acceleration were estimated by differentiating the velocity signals measured by the sensors. The frequencyweighted acceleration signals $a_{w}(t)$ were then obtained using the $W b$ weighting curve provided in BS 6841:1987 [40].

$$
\operatorname{VDV}=\left(\int_{0}^{T} a_{w}^{4}(t) d t\right)^{\frac{1}{4}}
$$

Where;

$$
a_{w}=\text { frequency-weighted acceleration }\left(\mathrm{m} / \mathrm{s}^{2}\right)
$$

It is clearly shown in Figure 20 that the highest VDV values are along corridor 2 (i.e. sensors 4, 5 and 6). On the other hand, the lowest VDV values correspond to the sensors of corridor 1 (i.e. sensors 1, 2 and 3). This observation is again consistent with the results of the trajectory-based 
floor busyness analysis depicted in Figure 16. This agreement also reveals the success of the individual video and vibration components of the monitoring system.

On another note, regarding the vibration levels within residential buildings, BS 6472-1:2008 standard [39] reports the VDV ranges that might result in adverse comments. For office floors, the standard suggests that there is a low probability of adverse comment for VDV values lower than $0.8 \mathrm{~m} / \mathrm{s}^{1.75}$. All VDVs shown in Figure 20 are well below this limit.

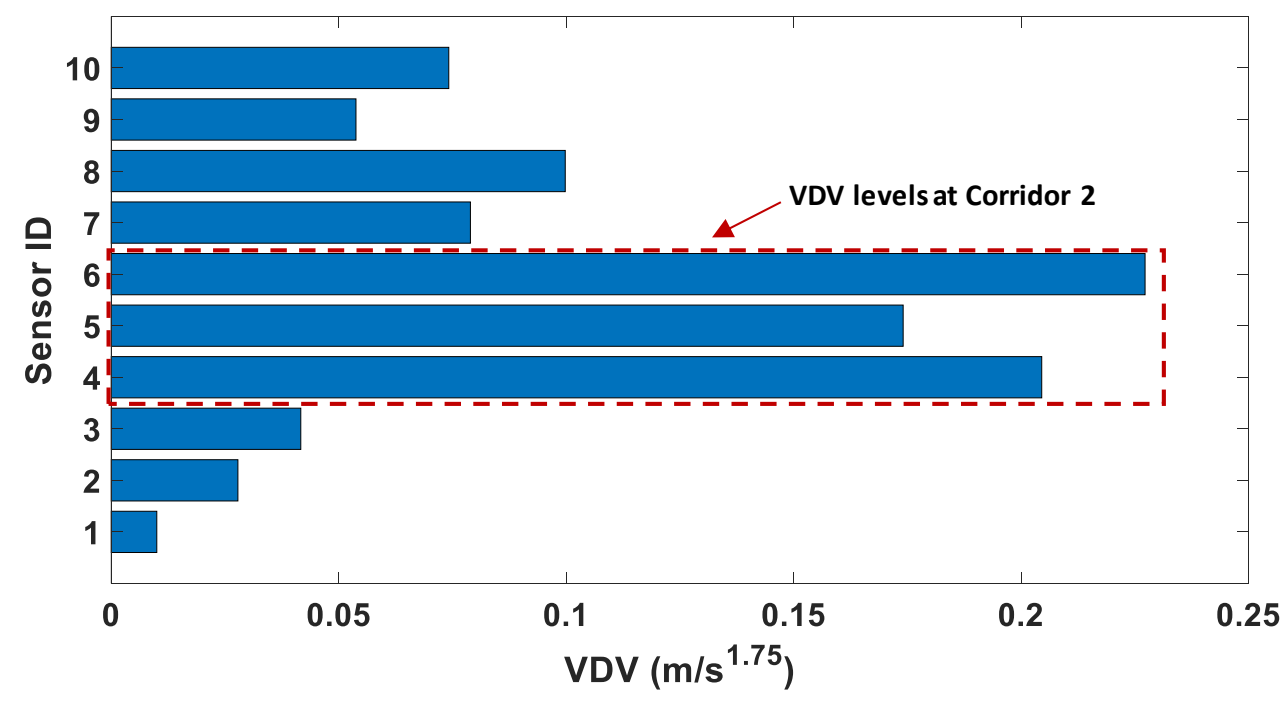

Figure 20. VDVs at the ten sensor locations.

Additionally, the response factor $(R)$ at each sensor location was calculated in accordance to SCI P354 [25]. It is to be noted that the moving RMS of the frequency-weighted acceleration signal $\left(a_{w, \mathrm{RMS}}\right)$ was computed using a 1 -sec RMS interval. The $R$ factor of each signal was then computed using Eq. (5), simply by taking the maximum value of $a_{w, \mathrm{RMS}}$ and normalizing it by $0.005 \mathrm{~m} / \mathrm{s}^{2}$. The maximum $R$ factors at the ten sensor locations are shown in Figure 21 .

$$
R=\frac{a_{w, \mathrm{RMS}}}{0.005 \mathrm{~m} / \mathrm{sec}^{2}}
$$


Where;

$$
a_{w, R M S}=\text { frequency-weighted RMS acceleration }\left(\mathrm{m} / \mathrm{s}^{2}\right)
$$

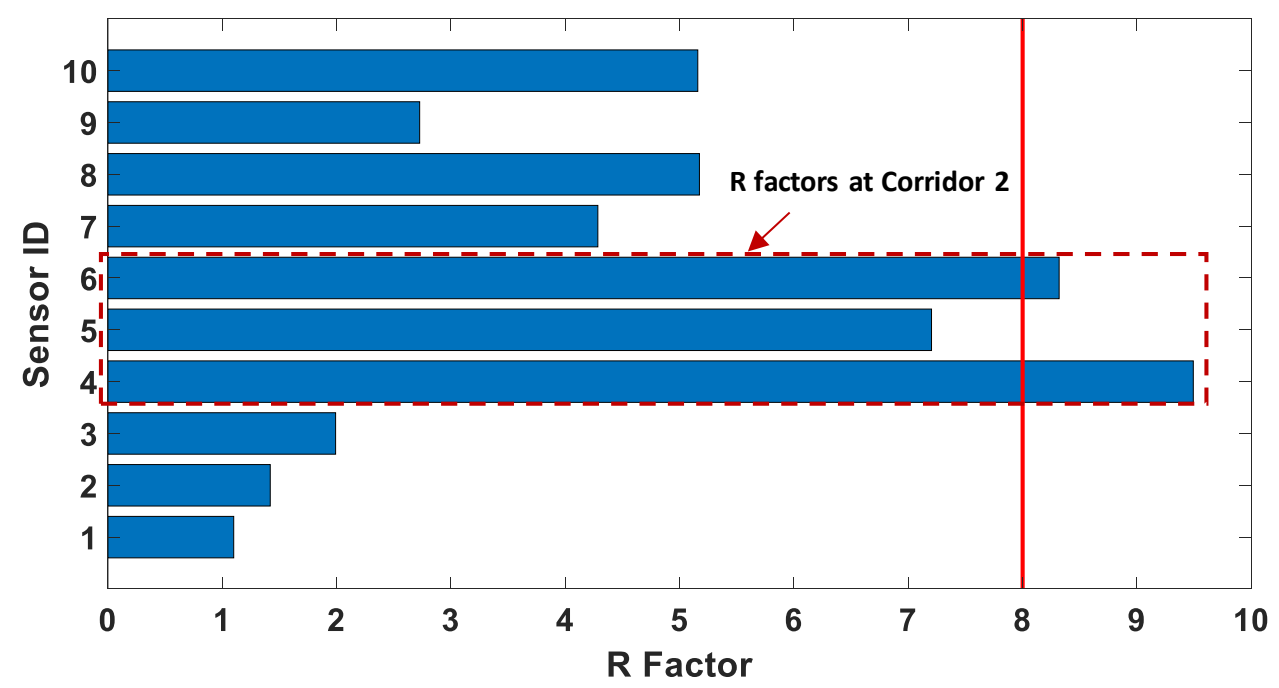

Figure 21. Maximum $\mathrm{R}$ factors at the ten sensor locations.

SCI P354 [25] has a recommended limit of $R=8$ for office floors. Based on Figure 21, the $R$ factors measured at the locations of sensors 4 and 6 are exceeding this limit very slightly, which indicates that excessive walking-induced excitations may result in complaints at these locations. However, it needs to be emphasized that the $R$ values are not very conclusive by small margin. It also needs to be mentioned that unlike the VDVs the maximum $R$ factor is not a function of busyness of the floor; therefore, the results of the Figure 21 cannot be compared to the heat map of Figure 16.

Overall, the trajectory analyses presented in this section indicate that the information extracted by the proposed video-vibration monitoring system can provide insight into the behavior of occupants on office floors. The system also showed great potential in analyzing the correlation between the walking patterns and the vibration response across the monitored floor. The outcome 
of the monitoring exercise described in this paper can be used to build a representative model for occupant movement on floors. One way of doing so is to use the extracted trajectories to train a data-driven crowd model [41] that produces simulated trajectories similar to the observed ones. Another approach would be to use the trajectories for fine-tuning the parameters of an agentbased crowd model [42].

\section{Conclusions}

This paper proposes an automated video-vibration monitoring system for identifying walking patterns and better understanding of occupant walking patterns in workplace environments. An office floor located in Doha, Qatar is utilized for data collection. Occupant tracking and detection algorithms are used to extract the walking trajectories from the recorded videos. The extracted trajectories are then analyzed to obtain useful information regarding the walking paths, pacing rates, and busyness of the floor with respect to time. The correlation between the measured vibration levels and the extracted trajectories was also investigated. The findings of this work

suggest that the proposed video-vibration monitoring system provides valuable information about the following:

- The activity of the floor with respect to time of the day.

- The busy spots of the monitored floor.

- The walking speeds inside office floors.

- How the occupants interact with each other and avoid obstacles.

- The relationship between the walking paths and vibration levels. 
Identifying all of these details is necessary for engineers to characterize vibration problems in existing office floors and propose efficient countermeasures.

In future studies, the proposed video-vibration monitoring system can be used to create an accurate model for predicting multi-person loading scenarios. This can be done with the following steps:

1. Use the proposed system to monitor a certain office floor.

2. Analyze the video recordings to extract occupant trajectories.

3. Analyze the trajectories to obtain several metrics that reflect the walking patterns of the occupants.

4. Develop a data-driven or agent-based crowd model according to the identified walking patterns. The metrics obtained from the analysis of the walking patterns can be used to fine tune the parameters of the crowd model and enable it to generate a large number of realistic multi-person walking scenarios. Each scenario includes a number of walking trajectories.

5. The trajectories associated with each walking scenario are used alongside a single occupant loading model (e.g. the inverted pendulum model of [43]) to create a large number of simulated multi-person loading scenarios.

6. Create an accurate finite element (FE) model of the tested floor.

7. Impose the simulated loading scenarios on the FE model.

8. The FE simulations can be used to perform a probabilistic assessment of the vibration levels of the floor. This exercise would allow the designer to predict vibration problems before they occur and/or it would enable the designer to evaluate vibration countermeasures before their actual implementation. 


\section{Acknowledgments}

The authors would like to thank Qatar Rail Company for providing an access to the office floor monitored in this work. The financial support for this research was provided by Qatar National Research Fund, QNRF (a member of Qatar Foundation) via the National Priorities Research Program (NPRP), Project Number: NPRP 8-836-2-353. The statements made herein are solely the responsibility of the authors.

\section{References}

[1] I.M. Díaz, E. Pereira, P. Reynolds, Integral resonant control scheme for cancelling humaninduced vibrations in light-weight pedestrian structures, Struct. Control Heal. Monit. (2012). doi:10.1002/stc.423.

[2] V. Racic, A. Pavic, J.M.W. Brownjohn, Experimental identification and analytical modelling of human walking forces: Literature review, J. Sound Vib. 326 (2009) 1-49. doi:10.1016/j.jsv.2009.04.020.

[3] S. Živanović, A. Pavic, P. Reynolds, Finite element modelling and updating of a lively footbridge: The complete process, J. Sound Vib. 301 (2007) 126-145.

doi:10.1016/j.jsv.2006.09.024.

[4] A. Barrett, O. Avci, M. Setareh, T.M. Murray, Observations from vibration testing of insitu structures, in: Proc., Struct. Congr. Expo. ASCE Struct. Congr., ASCE Reston, Va., 2006: pp. 1-10.

[5] S. Živanović, A. Pavić, P. Reynolds, Probability-based prediction of multi-mode vibration response to walking excitation, Eng. Struct. 29 (2007) 942-954.

doi:10.1016/j.engstruct.2006.07.004.

[6] E. Shahabpoor, A. Pavic, V. Racic, Structural vibration serviceability: New design framework featuring human-structure interaction, Eng. Struct. (2017). doi:10.1016/j.engstruct.2017.01.030.

[7] ASCE, Minimum Design Loads and Associated Criteria for Buildings and Other Structures, American Society of Civil Engineers, 2017. doi:10.1061/9780784414248.

[8] Z.O. Muhammad, P. Reynolds, E.J. Hudson, Evaluation of contemporary guidelines for floor vibration serviceability assessment, in: Conf. Proc. Soc. Exp. Mech. Ser., 2017. doi:10.1007/978-3-319-54777-0_42.

[9] O. Avci, Modal parameter variations due to joist bottom chord extension installations on laboratory footbridges, J. Perform. Constr. Facil. 29 (2015). doi:10.1061/(ASCE)CF.19435509.0000635 . 
[10] O. Avci, A. Bhargava, Y. Al-Smadi, J. Isenberg, Vibrations Serviceability of a Medical Facility Floor for Sensitive Equipment Replacement: Evaluation with Sparse In-Situ Data, Pract. Period. Struct. Des. Constr. (2018).

[11] T.M. Murray, Floor Vibrations: The Human Tolerance Side Of The Equation, 17th Int. Modal Anal. Conf. (1999).

[12] Z. Muhammad, P. Reynolds, O. Avci, M. Hussein, Review of Pedestrian Load Models for Vibration Serviceability Assessment of Floor Structures, Vibration. (2018). doi:10.3390/vibration2010001.

[13] O. Avci, Amplitude-dependent damping in vibration serviceability: Case of a laboratory footbridge, J. Archit. Eng. 22 (2016). doi:10.1061/(ASCE)AE.1943-5568.0000211.

[14] K. Van Nimmen, G. Lombaert, G. De Roeck, P. Van den Broeck, Vibration serviceability of footbridges: Evaluation of the current codes of practice, Eng. Struct. (2014). doi:10.1016/j.engstruct.2013.11.006.

[15] S. Zivanovic, A. Pavic, J.M.W. Brownjohn, Vibration serviceability assessment of slender structures using VSATs software, 26th Int. Modal Anal. Conf. (2008).

[16] K. Van Nimmen, G. Lombaert, G. De Roeck, P. Van den Broeck, Simulation of Humaninduced Vibrations Based on the Characterized In-field Pedestrian Behavior, J. Vis. Exp. (2016). doi:10.3791/53668.

[17] A. Devin, P.J. Fanning, A. Pavic, Nonstructural Partitions and Floor Vibration Serviceability, J. Archit. Eng. (2016). doi:10.1061/(ASCE)AE.1943-5568.0000171.

[18] V. Racic, J. Chen, Data-driven generator of stochastic dynamic loading due to people bouncing, Comput. Struct. (2015). doi:10.1016/j.compstruc.2015.04.013.

[19] V. Racic, J.B. Morin, Data-driven modelling of vertical dynamic excitation of bridges induced by people running, Mech. Syst. Signal Process. (2014). doi:10.1016/j.ymssp.2013.10.006.

[20] A. Younis, O. Avci, M. Hussein, B. Davis, P. Reynolds, Dynamic Forces Induced by a Single Pedestrian: A Literature Review, Appl. Mech. Rev. 69 (2017) 020802. doi:10.1115/1.4036327.

[21] E. Ahmadi, C. Caprani, S. Živanović, A. Heidarpour, Vertical ground reaction forces on rigid and vibrating surfaces for vibration serviceability assessment of structures, Eng. Struct. (2018). doi:10.1016/j.engstruct.2018.06.059.

[22] S. Živanović, A. Pavic, V. Racic, Towards modelling in-service pedestrian loading of floor structures, Conf. Proc. Soc. Exp. Mech. Ser. 1 (2012) 85-94. doi:10.1007/978-14614-2413-0_9.

[23] T.M. Murray, D.E. Allen, E.E. Ungar, D.B. Davis, Vibrations of Steel-Framed Structural Systems Due to Human Activity: Second Edition, American Institute of Steel Construction, 2016.

[24] A. Pavić, M. Willford, Vibration Serviceability of Post-Tensioned Concrete Floors, 
Append. G Post-Tensioned Concr. Floors Des. Handb. - Tech. Rep. 43. (2005) 99-107.

[25] A.L. Smith, S.J. Hicks, P.J. Devine, Design of floors for vibration - A new approach SCI P354, Revised Ed, Steel Constr. Institute, Ascot, Berkshire, U.K. SCI P354 (2009) 1-128. doi:10.13140/RG.2.2.29342.95048.

[26] M.R. Willford, P. Young, A design guide for footfall induced vibrations of structures, 2006.

[27] O. Avci, O. Abdeljaber, S. Kiranyaz, M. Hussein, D.J. Inman, Wireless and real-time structural damage detection: A novel decentralized method for wireless sensor networks, J. Sound Vib. 424 (2018). doi:10.1016/j.jsv.2018.03.008.

[28] O. Avci, O. Abdeljaber, S. Kiranyaz, D.J. Inman, Convolutional Neural Networks for Real-time and Wireless Damage Detection, in: IMAC XXXVII, Int. Modal Anal. Conf., Springer International Publishing, Orlando, FL, USA, 2019.

[29] The Mathworks Inc., MATLAB - MathWorks, (2016). doi:2016-11-26.

[30] F. Fleuret, J. Berclaz, R. Lengagne, P. Fua, Multicamera people tracking with a probabilistic occupancy map, IEEE Trans. Pattern Anal. Mach. Intell. 30 (2008) 267-282. doi:10.1109/TPAMI.2007.1174.

[31] J. Berclaz, A. Shahrokni, F. Fleuret, J. Ferryman, P. Fua, Evaluation of Probabilistic Occupancy Map People Detection for Surveillance Systems, Pets. (2009). doi:10.1021/la402417h.

[32] J. Berclaz, F. Fleuret, E. Türetken, P. Fua, Multiple object tracking using k-shortest paths optimization, IEEE Trans. Pattern Anal. Mach. Intell. (2011). doi:10.1109/TPAMI.2011.21.

[33] H. Ben Shitrit, J. Berclaz, F. Fleuret, P. Fua, Tracking multiple people under global appearance constraints, in: Proc. IEEE Int. Conf. Comput. Vis., 2011. doi:10.1109/ICCV.2011.6126235.

[34] H. Ben Shitrit, J. Berclaz, F. Fleuret, P. Fua, Multi-commodity network flow for tracking multiple people, IEEE Trans. Pattern Anal. Mach. Intell. (2014). doi:10.1109/TPAMI.2013.210.

[35] F. Fleuret, H. Ben Shitrit, P. Fua, Re-identification for improved people tracking, in: Adv. Comput. Vis. Pattern Recognit., 2014. doi:10.1007/978-1-4471-6296-4_15.

[36] T. Bagautdinov, F. Fleuret, P. Fua, Probability occupancy maps for occluded depth images, in: Proc. IEEE Comput. Soc. Conf. Comput. Vis. Pattern Recognit., 2015. doi:10.1109/CVPR.2015.7298900.

[37] R. Szeliski, Computer Vision: Algorithms and Applications, Springer, 2011. doi:10.1007/978-1-84882-935-0.

[38] Z. Zivkovic, F. Van Der Heijden, Efficient adaptive density estimation per image pixel for the task of background subtraction, Pattern Recognit. Lett. 27 (2006) 773-780. doi:10.1016/j.patrec.2005.11.005. 
[39] British Standards, BS 6472-1:2008 Guide to evaluation of human exposure to vibration in buildings. Vibration sources other than blasting., 2008.

[40] British Standards Institution, BS 6841:1987 Guide to measurement and evaluation of human exposure to whole-body mechanical vibration and repeated shock, BSI. (1987).

[41] J. Zhong, W. Cai, L. Luo, H. Yin, Learning Behavior Patterns from Video : A Data-driven Framework for Agent-based Crowd Modeling, in: Int. Conf. Auton. Agents Multiagent Syst., 2015: pp. 801-809.

[42] C.M. Henein, T. White, Agent-Based Modelling of Forces in Crowds, (2010) 173-184. doi:10.1007/978-3-540-32243-6_14.

[43] M. Bocian, J.H.G. Macdonald, J.F. Burn, Biomechanically Inspired Modeling of Pedestrian-Induced Vertical Self-Excited Forces, J. Bridg. Eng. (2013). doi:10.1061/(asce)be.1943-5592.0000490. 